La théorie quantique est le socle sur lequel repose notre compréhension de

la nature. Bien qu'elle ait été élaborée il y a presque un siècle, l'établissement expérimental de ses fondements se poursuit. En particulier,

l'exploration des aspects

quantiques du transport de chaleur n'en est qu'à ses débuts. Nous présentons ici

la démonstration expérimentale d'une des pierres angulaires du transport de la chaleur en régime quantique, l'existence d'une limite maximum

fondamentale à la conduction thermique appelée, pour un canal élémentaire de transport, le quantum de conductance thermique.

Ce quantum, ici mesuré dans un système électronique et égal à 1 pW/K à une température de $1 \mathrm{~K}$, est prédit complètement universel, indépendant même du mécanisme de conduction thermique.

\title{
Limite quantique du flux de chaleur
}

Anne Anthore (anne.anthore@univ-paris-diderot.fr) Sébastien Jézouin, François Parmentier, Ulf Gennser et Frédéric Pierre (frederic.pierre@|pn.cnrs.fr)

Laboratoire de photonique et de nanostructures, LPN-CNRS, Route de Nozay, 91460 Marcoussis

\section{Quelques définitions}

\section{La chaleur}

Parmi les différentes formes d'énergie, la chaleur est celle qui a le plus questionné les scientifiques. Pendant longtemps, la chaleur a été perçue comme un fluide d'une nature inconnue, le calorique. Des expériences décisives, réalisées par James Prescott Joule entre 1840 et 1843, permirent de démontrer qu'elle n'était qu'un transfert d'énergie, équivalent au travail mécanique ou au travail électrique.

La problématique de la chaleur connât depuis le début du $\mathrm{XXI}^{\mathrm{e}}$ siècle un regain d'intérêt considérable. En effet, la gestion de la chaleur est cruciale pour les technologies de l'information et de la communication, et une meilleure exploitation des phénomènes thermoélectriques permettrait d'obtenir de nouvelles sources d'énergie. D'un point de vue fondamental, avec les progrès de la miniaturisation des composants électroniques, il est désormais possible d'atteindre un régime où le transport de chaleur est régi par la mécanique quantique. Ceci ouvre la voie à un nouveau champ d'investigation : que sont les phénomènes thermiques quantiques ? Peut-on réaliser de nouvelles machines thermiques basées sur la manipulation quantique de la chaleur?

\section{Les conducteurs quantiques}

La mécanique quantique décrit la matière à la fois comme ondes et comme particules. Un conducteur quantique est un conducteur (électrique, de chaleur...) dont les propriétés préservent des traces de la nature ondulatoire des particules de conduction. En conséquence, malgré la diversité de ces particules (électrons, excitations électroniques plus exotiques comme les paires électron-trou, photons, phonons...), tout conducteur quantique reliant deux réservoirs de particules aux propriétés thermodynamiques différentes s'apparente à un guide d'onde unidimensionnel constitué de plusieurs modes élémentaires discrets, appelés " canaux de conduction ". Pour des petits conducteurs quantiques, lorsque les interactions coulombiennes peuvent être ignorées, les différents canaux sont indépendants les uns des autres. Dans cette limite, comprendre la conduction à l'échelle élémentaire d'un canal permet alors de décrire n'importe quel conducteur quantique plus complexe.

En pratique, on peut avoir affaire à un conducteur quantique à température ambiante si toutes ses dimensions sont très petites, de l'ordre du nanomètre. Depuis les années 1980, avec notamment les progrès en nanofabrication par lithographie électronique, il est possible de réaliser des conducteurs électroniques, photoniques ou phononiques avec une résolution de $10 \mathrm{~nm}$. Pour étudier le comportement quantique de ces conducteurs dont la taille varie entre quelques dizaines de nanomètres et quelques microns, les expériences sont réalisées à basse température, typiquement en dessous de quelques kelvins. L'étude fondamentale du transport quantique prépare aussi la future nanoélectronique, qui sera certainement quantique.

\section{Limites des flux d'électricité et de chaleur dans les conducteurs quantiques}

Une des prédictions de la mécanique quantique est que le flux maximum de charges pouvant s'écouler dans un unique canal de conduction électronique est fondamentalement limité. Ce qui, en général, se traduit par une borne maximale $G_{e}$ à la conductance électrique d'un tel canal (voir encadré), appelée « quantum de 


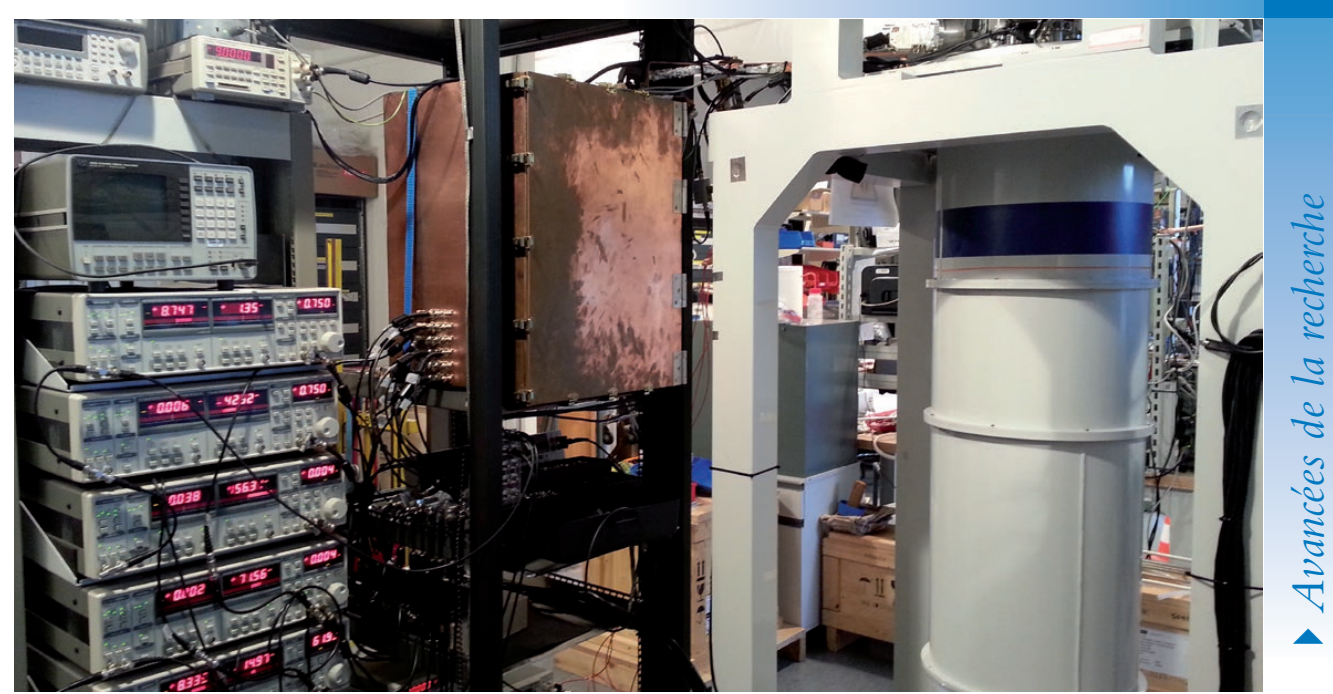

Système de mesure ultra sensible centré autour d’un réfrigérateur à dilution, au Laboratoire de photonique et de nanostructures (LPN-CNRS), à Marcoussis (91).

conductance électrique ». Autrement dit, le plus simple des conducteurs quantiques, ne comportant qu'un seul canal de conduction électronique, a une résistance électrique minimum non nulle $1 / G_{e}=h / e^{2} \sim 26$ kilo-ohms, quelle que soit la façon dont il est réalisé (matériau, géométrie). En effet, cette valeur de $G_{e}$ ne dépend que de la constante de Planck $h$ et de la charge de l'électron $e$. Cette limite a d'abord été démontrée expérimentalement dans des matériaux semi-conducteurs au milieu des années 80 . Depuis, la même limite de conductance électrique a été mesurée dans des métaux et des matériaux organiques, montrant ainsi son caractère universel. Cependant, la valeur du quantum de conductance électrique est liée au quantum de charge transmis d'un côté à l'autre du canal de conduction. Il peut donc être différent selon la nature des particules électroniques mises en jeu, et notamment dans le régime de l'effet Hall quantique fractionnaire.

Généralement, un bon conducteur d'électricité est aussi un bon conducteur de chaleur. En effet, les porteurs de charge véhiculent également de la chaleur : le courant électrique est lié à leurs charges, alors que le courant de chaleur est lié à leurs énergies. Pour les même raisons fondamentales que le flux d'électricité, le flux de chaleur dans un unique canal de conduction électronique est limité et la conductance thermique a un maximum appelé « quantum de conductance thermique ", qui ne dépend que de la constante de Boltzmann $k_{B}$, de la constante de Planck $h$ et de la température moyenne des réservoirs (voir encadré). Il existe aussi des conducteurs de chaleur qui ne conduisent pas l'électricité. Dans ce cas, les « particules " impliquées dans les transferts de chaleur peuvent être les phonons ou les photons. De façon remarquable, la valeur trouvée pour le quantum de conductance

\section{Quantum de conductance électrique et thermique} dans un canal unique de conduction électronique

Lorsqu'une différence de potentiel électrique ou de température entre les réservoirs est appliquée aux bornes du canal de conduction de la figure E1a, la conduction électrique ou de chaleur est régie par la mécanique quantique. Ceci signifie que la conduction se fait par des "particules», dont les propriétés respectent le principe d'incertitude temps-énergie de Heisenberg. Dans une représentation temps-énergie (fig. E1b), la charge ou la chaleur sont transmises par des "particules » correspondant à des paquets d'aire minimale $\Delta E . \Delta t=h$ (zone grisée de la figure $E 1 b$ ). Pendant un temps $t$, pour une énergie fournie $E$, le nombre maximum de paquets transmis est $N=E t / h$. Ce nombre permet de déterminer les conductances thermique et électrique maximales obtenues pour un conducteur quantique parfait, dit balistique. Dans le cas de la conduction électrique, l'énergie $E=\mathrm{eV}=\mathrm{e}\left(V_{L}-V_{R}\right)$ est fournie par le générateur, où e est la charge de l'électron. Chaque paquet portant la charge e, le courant électrique maximal résultant est $I=\mathrm{eN} / \mathrm{t}=\mathrm{e}^{2} \mathrm{~V} / \mathrm{h}$. La conductance électrique dans ce régime linéaire est alors bornée au maximum à $G_{\mathrm{e}}=I / \mathrm{V}=\mathrm{e}^{2} / \mathrm{h}$.

Dans le cas de la conduction de chaleur, chaque réservoir ( $L$ ou $R$ ) met à disposition une énergie de l'ordre de $k_{B} T$, où $k_{B}$ est la constante de Boltzmann et $T=T_{L, R}$ la température du réservoir. Le flux de chaleur sortant d'un réservoir est alors $J_{L, R}=N_{L, R} \alpha k_{B} T_{L, R} / t$, où $\alpha$ est un facteur d'ordre 1 et $N_{L, R}$ est le nombre de paquets transmis par le réservoir $L$ (ou $R$ ). En substituant $N_{L, R}$ par $k_{B} T_{L, R} t / h$, les flux de chaleur émis par chaque réservoir s'écrivent $J_{L, R}=\alpha\left(k_{B} T_{L, R}\right)^{2} / h$, et le courant de chaleur total est $J=J_{L}-J_{R}=\alpha k_{B}^{2}\left(T_{L}^{2}-T_{R}^{2}\right) / h$. On peut montrer [1] que $\alpha=\pi^{2} / 6$, quelle que soit la particule transportant la chaleur. Le quantum de conductance thermique vérifie donc $G_{t h}=J /\left(T_{L}-T_{R}\right)=\pi^{2} k_{B}^{2}\left(T_{L}+T_{R}\right) / 6 h$.

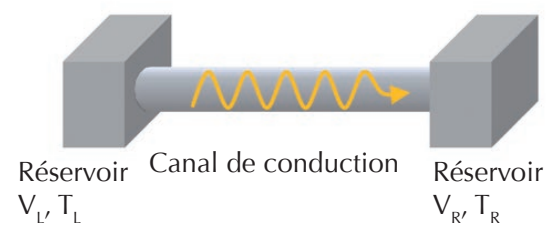

$\mathrm{a}$

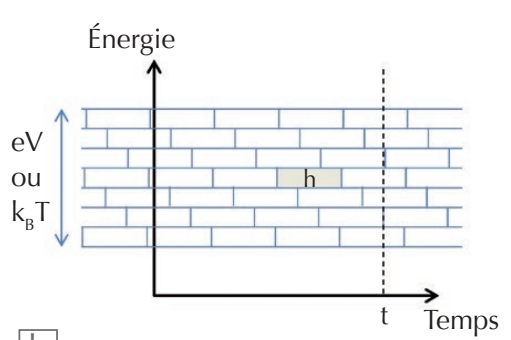

$\mathrm{b}$
E1. (a) Modélisation d'un canal unique de conduction entre deux réservoirs thermodynamiques $L$ et $R$, de températures $T_{L}$ et $T_{R}$ et de potentiels électriques $V_{L}$ et $V_{R}$ : une onde se propage entre les deux réservoirs, résultant en un transfert d'énergie entre les deux.

(b) Représentation temps-énergie de " particules » transmettant l'énergie dans un unique canal de conduction. L'aire minimale du paquet grisé est la constante de Planck $h$, d'après le principe d'incertitude de Heisenberg. 
>>

thermique est la même pour toutes ces "particules".

En raison du lien étroit entre chaleur, entropie et information, des travaux théoriques cherchent à démontrer que cette universalité du quantum de conductance thermique, quel que soit le matériau et quelle que soit la "particule » de conduction, est liée à une limite fondamentale du flux maximum de transfert d'information dans un unique canal de conduction quantique.

\section{Détermination expérimentale de la limite quantique du flux de chaleur}

Contrairement au courant électrique, le courant de chaleur ne se mesure pas à l'aide d'un simple appareil. Pour le déterminer, il faut mesurer des différences de température liées à des petits transferts d'énergie bien maitrisés. Pour atteindre le quantum de conductance thermique, il faut de plus que tous les canaux de conduction élémentaires soient parfaits. Ce niveau de contrôle n'est possible que si leur nombre et, en conséquence, le courant de chaleur sont très petits. Le quantum de conductance thermique, égal à $1 \mathrm{pW} / \mathrm{K}$ à une température de $1 \mathrm{~K}$, n'a donc été confirmé expérimentalement que relativement récemment et uniquement pour des phonons (modes de vibration du solide) et pour des photons (modes électromagnétiques). Pour les électrons, seule une estimation d'ordre de grandeur avait pu être réalisée [1].

Nous avons, pour la première fois, déterminé la limite quantique du flux de chaleur dans un unique canal de conduction électronique, et déduit la valeur du quantum de conductance thermique avec une précision de l'ordre du pourcent [2]. Pour parvenir à ce résultat, nous avons injecté quelques femtowatts $\left(10^{-15} \mathrm{~W}\right)$ de puissance électrique dans une électrode métallique micrométrique (représentée en rouge sur la figure 1 et appelée microélectrode dans la suite). À des températures de quelques centièmes de degrés au-dessus du zéro absolu $\left(-273,14^{\circ} \mathrm{C}\right)$, la puissance injectée chauffe légèrement la microélectrode et est principalement évacuée par un petit nombre de canaux de conduction électronique parfaitement balistiques, qui peuvent être ouverts ou fermés in situ (fig. 1). La température dans la microélectrode est mesurée très précisément à partir des fluctuations de courant électrique associées. La limite quantique du flux de chaleur est alors

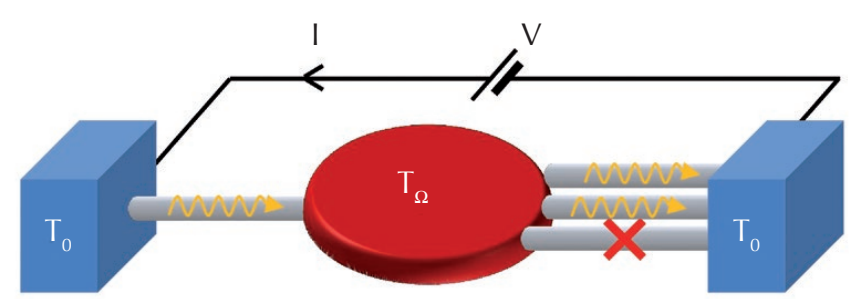

a

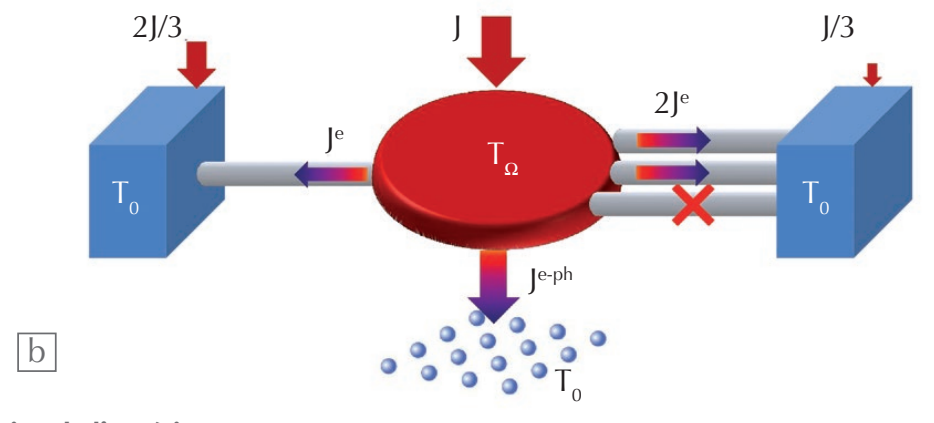

\section{Principe de l'expérience}

(a) Modélisation de l'expérience en terme de réservoirs et de canaux de conduction quantiques électroniques. Une tension électrique $V$ est appliquée entre deux électrodes métalliques jouant le rôle de réservoirs électroniques froids (bleus). La taille millimétrique des réservoirs bleus est telle que malgré la puissance électrique injectée (2J/3 et J/3 pour la configuration représentée ici), la température électronique reste constante et égale à la température $T_{0}$ de l'environnement de l'échantillon. Le courant électrique / s'écoule au travers d'un nombre ajustable $n$ de canaux de conduction électronique (en gris, ici trois sont ouverts, un est fermé), en passant par un réservoir intermédiaire métallique de taille micrométrique (" microélectrode » rouge, l'échelle n’est pas respectée). De par sa taille, les électrons de la microélectrode seront légèrement chauffés par effet Joule jusqu'à une température $T_{\Omega}$ (b) Bilan des flux de chaleur en régime stationnaire. En régime stationnaire, la température $T_{\Omega}$ sera déterminée par l'équilibre entre les flux de chaleur entrant et sortant de la microélectrode rouge. Ce réservoir est chauffé par l'injection d'une puissance Joule électrique connue $J=$ VI/2 (le facteur 1/2 par rapport à la puissance Joule totale provient du fait que l'autre moitié de cette puissance est dissipée dans les réservoirs bleus). La chaleur s'évacue par un nombre ajustable $n$ de canaux de conduction électronique (flux $3 J^{e}$ ici) vers les réservoirs électroniques froids (bleus) et très légèrement vers les phonons de la microélectrode (flux $J^{e-p h}$ ). Aux basses températures de l'expérience, les phonons de la microélectrode sont fortement découplés des électrons et en conséquence froids.

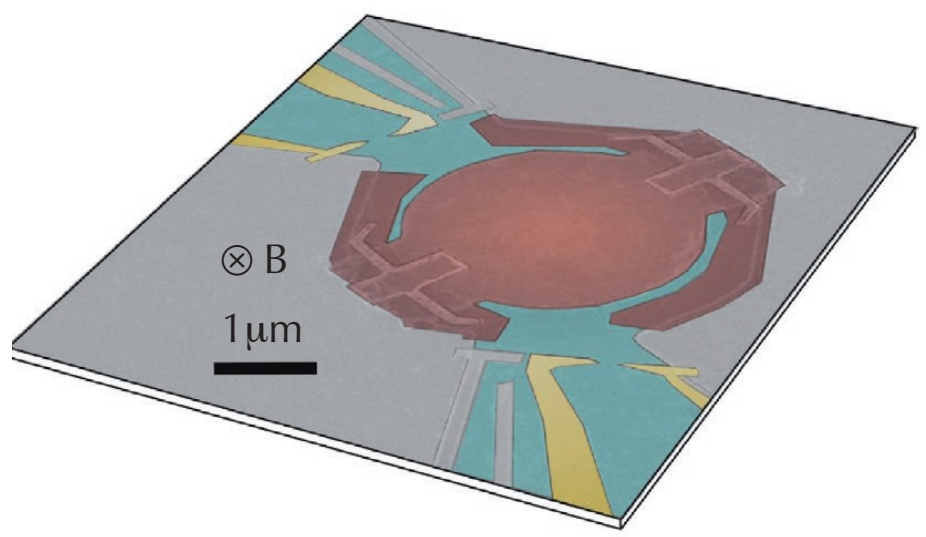

2. Image coloriée, obtenue au microscope électronique à balayage, de l'échantillon utilisé dans l'expérience et fabriqué à partir de matériaux semi-conducteurs (zone cyan). L'électrode métallique chauffée, appelée microélectrode, est la partie coloriée en marron. Les pointes jaunes permettent de définir dans l'empilement de semi-conducteurs, des conducteurs quantiques électroniques dont le nombre de canaux de conduction est ajustable par l'application d'une simple tension électrique. Les réservoirs froids ne sont pas visibles à cette échelle. L'échantillon est placé dans un réfrigérateur à dilution, sous vide, à une température de 0,02 K (photo p. 17), et soumis à un champ magnétique perpendiculaire $\mathbf{B}$ pour atteindre précisément le régime balistique. 


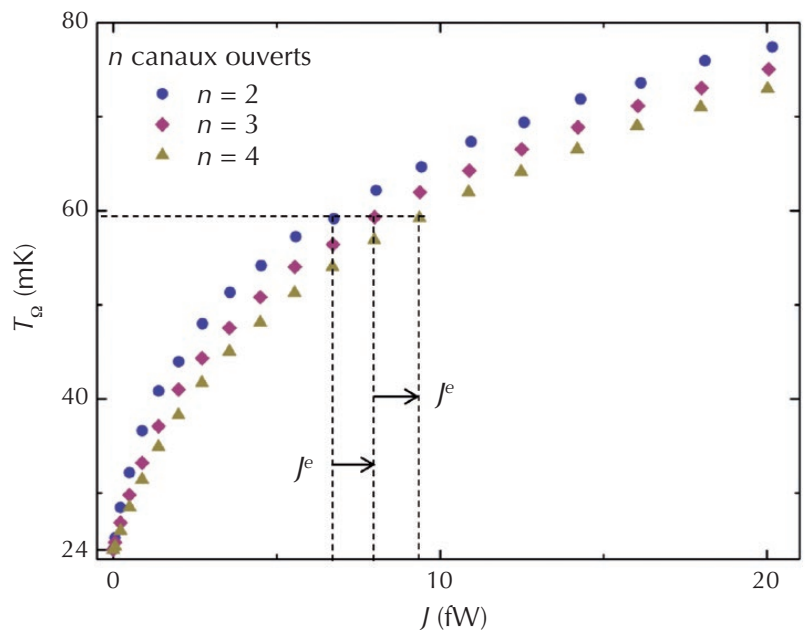

3. Température $T_{\Omega}$ de la microélectrode en fonction de la puissance Joule $J$ injectée pour 2, 3 ou 4 canaux de conduction ouverts. À une température donnée $T_{\Omega}$, le flux de chaleur $f^{e}$ circulant dans un seul canal de conduction électronique est déduit de la différence de puissance injectée $J$ entre les configurations à $n-1$ canaux ouverts et $n$ canaux ouverts.

déterminée par la quantité de puissance supplémentaire à injecter pour maintenir la température de la microélectrode constante lorsqu'un canal de conduction additionnel est ouvert (fig. 1).

Bien que le principe de l'expérience soit simple et direct, il est nécessaire de maîtriser deux points cruciaux pour la mettre en œuvre : il faut connaitre très précisément la puissance injectée dans la microélectrode chauffée et mesurer des températures au millikelvin près. Ceci a pu être réalisé grâce aux mêmes matériaux semi-conducteurs et à la même façon de réaliser les conducteurs quantiques que dans les expériences pionnières ayant mesuré le quantum de conductance électrique (fig. 2) (a). En effet, l'exceptionnelle qualité cristalline de ces matériaux permet d'atteindre le régime dit balistique dans lequel le transport électrique et de chaleur atteint la limite quantique, et la modularité par effet de champ des semiconducteurs permet de contrôler in situ le nombre de canaux de conduction. Ainsi, en appliquant une tension électrique $V$ aux bornes de l'échantillon et connaissant exactement sa conductance électrique totale, la puissance Joule injectée est déduite avec une précision de quelque centièmes de femtowatt. Dans un conducteur balistique, la chute de potentiel électrique a lieu à l'interface entre les réservoirs et le canal de conduction. La puissance électrique est donc uniquement dissipée dans les réservoirs et non pas dans le canal de conduction. L'effet du chauffage des électrons par effet
Joule est négligeable dans les réservoirs de grande taille (bleus) et non négligeable dans la microélectrode métallique de petite taille (rouge). Le couplage entre les électrons et les phonons froids, proportionnel au volume du réservoir, est responsable de cette différence de comportement. Ceci est vérifié précisément en mesurant la température de tous les réservoirs à partir des fluctuations de courant électrique résultant de l'agitation thermique des électrons dans les réservoirs. L'amplitude des fluctuations étant très petite (de l'ordre du femtoampère) aux très basses températures de l'expérience, nous avons développé au laboratoire des amplificateurs de tension cryogéniques ultrasensibles qui nous ont permis d'obtenir une résolution meilleure que le millikelvin.

En pratique, nous mesurons la température $T_{\Omega}$ de la microélectrode en fonction de la puissance Joule injectée $J$ pour différents nombres de canaux de conduction ouverts (fig. 3). Plus il y a de canaux ouverts moins la température de la microélectrode est élevée, car la chaleur a plus de chemins électroniques pour s'évacuer. À une température $T_{\Omega}$ de la microélectrode fixée, le flux de chaleur s'évacuant par un unique canal de conduction est la différence entre les puissances injectées pour obtenir cette température avec $n$ canaux ouverts et $n-1$ canaux ouverts. En faisant cette différence, nous nous affranchissons de la petite contribution parasite de l'évacuation de chaleur de la microélectrode métallique vers les phonons froids (qui dépend uniquement de la température, et pas du nombre de canaux électroniques ouverts ; l'évacuation de chaleur vers les phonons au niveau des canaux de conduction est complètement négligeable, de par leur très petite taille). Nous obtenons ensuite le flux de chaleur par canal de conduction électronique en fonction de la température et pouvons vérifier qu'il dépend, comme attendu, de la différence des carrés des températures des réservoirs. En étudiant l'évolution du flux de chaleur électronique en fonction du nombre $n$ de canaux de conduction électronique ouverts, nous avons pu déduire la valeur du quantum de conductance thermique pour un unique canal de conduction et constater qu'elle correspondait à quelques pourcents près à sa valeur limite $G_{K}=\left(\pi^{2} / 3\right)\left(k_{B}^{2} / h\right) T=T p W / K^{2}$.

\section{Conclusion}

Nous avons déterminé expérimentalement la valeur du quantum de conductance thermique qui, de manière analogue au quantum de conductance électrique, donne l'échelle caractéristique des phénomènes thermiques quantiques. La résolution atteinte, de l'ordre du pourcent, ouvre ainsi la voie à l'exploration de nouveaux dispositifs basés sur la manipulation quantique des flux de chaleur. En particulier, les interférences quantiques entre différents chemins pour le courant de chaleur permettent de réaliser l'équivalent thermique de l'interféromètre optique. D'un point de vue fondamental, le parfait accord obtenu avec la valeur prédite pour le quantum de conductance thermique établit expérimentalement un des fondements principaux de la théorie quantique du transport de chaleur.

(a) Un gaz bidimensionnel d'électrons est créé à l'interface entre l'arséniure de gallium pur et l'arséniure de gallium substitué à 33\% par de l'aluminium. Par effet de champ avec des grilles métalliques polarisées en tension, les électrons du gaz sont confinés latéralement pour réaliser un contact ponctuel quantique, conducteur quantique modèle pouvant présenter jusqu'à une dizaine de canaux de conduction.

\section{Références}

1• Y. Dubi et M. Di Ventra, “Colloquium: Heat flow and thermoelectricity in atomic and molecular junctions", Reviews of Modern Physics 83 (2011) 131.

2• S. Jezouin, F.D. Parmentier, A. Anthore, U. Gennser, A. Cavanna, Y. Jin et F. Pierre, "Quantum Limit of Heat Flow Across a Single Electronic Channel", Science 342 (2013) 601. 